\title{
Cellulose-Affinity Isolation of Vaccine Candidate Antigens from Transgenic Plants
}

\author{
HMM Tariq Hossain ${ }^{1,}$, Kwan Yong Choi ${ }^{2}$ \\ ${ }^{1}$ Department of Agronomy, Sher-e-Bangla Agricultural University, Dhaka, Bangladesh \\ ${ }^{2}$ Department of Convergence Biotechnology, Pohang University of Science and Technology, Pohang, Korea
}

Email address:

hmmtariq@yahoo.com (HMM T. Hossain),kchoi@postech.ac.kr (K. Y. Choi)

${ }^{*}$ Corresponding author

\section{To cite this article:}

HMM Tariq Hossain, Kwan Yong Choi. Cellulose-Affinity Isolation of Vaccine Candidate Antigens from Transgenic Plants. Chemical and Biomolecular Engineering. Vol. 3, No. 1, 2018, pp. 1-10. doi: 10.11648/j.cbe.20180301.11

Received: January 31, 2017; Accepted: December 7, 2017; Published: January 2, 2018

\begin{abstract}
Apart from tradition, the biospecific affinity isolation has become one of the most rapidly growing concerns with cellulose binding domains (CBDs), a high-capacity tag for cost-effective purifications of fusion proteins. We report here a new strategy optimized for isolation of two fusion proteins, the FGF-1 (a human functional protein) and the $\mathrm{H}_{5} \mathrm{~N}_{1}$ (a vaccine candidate antigen) tagged with CBD were grown in transient Nicotiana benthamiana and transgenic Arabidopsis thaliana respectively. A notable fraction of the recombinant proteins was lost through plant debris pelleted from the plant-slurry made. However, this issue was resolved by adjusting tissue-to-buffer ratios with 1:10 and 1:15 in those plants respectively. Washing efficiencies were improved by agitating column beds with acidic buffer (20mM NaAc. pH 4.0) in Nicotiana and alkaline buffer (10mM Tris-base pH 8.0) in Arabidopsis. Adsorption and coupling of tagged proteins on cellulose matrices were affected by the buffer-logged resins. The column-beds, after pumping the moisture out, showed efficient in binding of antigens with almost no losses detected by immunoblot signals. The bound antigens were released efficiently from the cellulose matrices by $1 \%$ Cellobiose and $2 \%$ Triethylamine respectively. The successive purifications of these antigenic proteins with identical tags likely indicate the efficiency of the proposed strategy in providing a generic and cost-effective method to purify fusion proteins propagated in transgenic plants.
\end{abstract}

Keywords: CBD Tag, Vaccine Antigens, Affinity Separation, Plant

\section{Introduction}

Till date, the use of plants as green bioreactors has been the most technical breakthrough in heterologous gene expression rendered with recombinant production of novel bioactive molecules (e.g., antigenic proteins, antibodies, vaccines). Traditionally, recombinant proteins with medicinal properties have been synthesized and isolated by microbial fermentation, mammalian cell culture, or through transgenic animals. However, using transformed plants as platforms for propagating recombinant proteins have proved much more beneficial in comparison to other already established practices [1]. Plants engineered with genes encoding antigenic proteins of various pathogenic, viral, and bacterial organisms have been shown to correctly express the proteins that elicit production of antibodies in mammalian hosts [2]. Notably the cell biological configuration and some endogenous organelles equipped with supporting protein machinery in complex protein trafficking pathways facilitate the correct post-translational processing of foreign proteins in plants. As such, plants can readily and correctly handle the polypeptide backbone in expressing, folding, assembling, and glycosylation, towards fidelity of antigenic proteins very similar to animal cells [3].

Propagation of recombinant proteins through transgenic plants offers inexpensive initial running costs with the ease of scaling up [4]. Additionally, plant systems have a low risk of toxic contamination because they are free from infectious agents (such as virions and prions), oncogenic DNA sequences, and endotoxins [2,5]. Endotoxins can induce pyrogenic reactions and shock in mammals upon intravenous injection at levels as low as $1 \mathrm{ng} / \mathrm{mL}$ [5]. For instance, recombinant proteins produced from Escherichia coli are contaminated with endotoxins, the removal of which requires 
additional costs and time [7, 8]. Contrastingly, plant-based vaccines or plantibodies (antibodies) are fairly attractive because plants donot harbor human pathogens thus reducing the extra costs for screening viruses and bacterial toxins [9]. Using plant biosynthates as antibodies [10] and sub-unit vaccines [11] has turned out to be very efficient and has led to the first clinical trial of medicinal polypeptides, of which one quarter are antigenic proteins [12]. However, there are several challenges associated with the recombinant production of bio-molecules in plants; the selection of plant systems for commercial-scale production of target molecules through cost effective purification is one such technical bottleneck that needs to be overcome [13].

Purification of recombinant protein products in plants or fermentation broths rely mainly on commercial chromatographic systems, which are highly expensive in vaccine biotechnology. The efficacy of product purification systems is dependent on the downstream steps of the biomanufacturing process for selective isolation of the product by affinity chromatography [14]. Irrespective of the production system and precision level desired, downstream processing comprises upto $80 \%$ of overall target production costs [15]. Nevertheless, affinity separation represents an important tool to capture tagged proteins, with high resolution of purity, through selective separation of micro and macro plant molecules in solution. In this regard, cellulose binding domains (CBDs) have the potential to be used as an affinity tag like poly-histidine, glutathione Stransferase, and maltose binding proteins, which are being considered as coupling partners for easy isolation of molecules [16-18]. However, the inherent costs of the affinity-chromatographic systems appear as critical barriers particularly in preparative-scale preparations [19]. As such, a cheap and powerful system, with the opportunity of purification, aided by the affinity tag co-expressed with the byproduct of interest is desirable [20-21]. In this case, protein separation aided by the CBD tags is clearly attractive because it can be fused genetically with a target protein to facilitate specific sticking with cheap and versatile cellulose matrices or particles. Thus, the purification system can potentially handle large clarified sample volumes. It minimizes multistep purification with selective adsorption on cellulose particles, an available material with low price and low nonspecific affinity for most proteins [22]. Due to the potential affinity of CBDs to cellulose support matrices, this system can provide robust and highly regulated, pure concentrations of recombinant target proteins [23].

We tested the purification system emphasizing the process for total initial recovery of the target proteins and their affinity adsorption on the modified microcrystalline cellulose (MMC) under varying buffer conditions. Since the major cost is reflected in isolation and purification but not from recombinant production of the proteins in plants per se [24$25]$, we describe here the protocol for maximization of initial recovery of the target compounds from harvested plant biomass into the clarified homogenates of wild tobacco (Nicotiana benthamiana) and Arabidopsis (Arabidopsis thaliana). The CBD fused proteins $\left(\mathrm{FGF}_{1}\right.$ and $\left.\mathrm{H}_{5} \mathrm{~N}_{1}\right)$ were designed to deposit at endoplasmic reticulum (ER) of given plant cells and accumulated to around $2 \%$ of total soluble protein (TSP), a common benchmark cited for commercial molecular farming [26-27]. Considering the lower deposition of the target product per unit biomass, we explored the use of an inexpensive purifying system that could efficiently capture tagged proteins grown in genetically modified plants.

\section{Materials and Methods}

\subsection{Transient Transformation of Nicotiana benthamiana $L$. for $\mathrm{FGF}_{1}-\mathrm{CBD}$}

\section{Protein}

A constructed binary plasmid with recombinant protein DNA insert and a suppressor plasmid without insert were introduced separately into the agrobacterial strain (GV3101). The bacterial inocula carrying the binary plasmids were grown overnight at $28^{\circ} \mathrm{C}$ in $\mathrm{LB}$ medium $(5 \mathrm{~mL})$ with antibiotics $(50 \mu \mathrm{g} / \mathrm{mL}$ Kanamycin and $50 \mu \mathrm{g} / \mathrm{mL}$ Rifampicin). The total cultures were added to infect $100 \mathrm{~mL}$ of the LB medium supplemented with $20 \mu \mathrm{M}$ acetosyringone plus antibiotics (as above) and grown upto the $\mathrm{OD}_{600} 1.5-2.0$. An equal volume of both cultures $(1: 1)$ were mixed in an infiltration solution containing $\mathrm{MgCl}_{2}$ and acetosyringone [28]. The cultures were maintained in an ice-bath condition till infiltration was started. The top two to three leaves of plants were infiltrated with the bacterial solution using a $1 \mathrm{~mL}$ syringe without a needle. The infiltration solution was gently pushed into the leaves and the progress of the process was followed by a slight change in color in the infiltrated areas. The leaves were harvested after 3 days of infiltration and stored at $-80^{\circ} \mathrm{C}$ in plastic freezer bags.

\subsection{Propagation of Recombinant Proteins}

\subsection{1. $\mathrm{H}_{5} \mathrm{~N}_{1}-\mathrm{CBD}$ Protein in Transgenic Arabidopsis}

Transgenic seeds of Arabidopsis thaliana, at $2 \mathrm{~cm}$ and $3 \mathrm{~cm}$ seed to seed and row to row distances, respectively, were sown in plastic trays $(55 \mathrm{~cm} / 25 \mathrm{~cm} / 4 \mathrm{~cm})$ filled with vermiculite and bioplaque $(1: 1)$. The plantlets were gown in plant growth room maintaining 12-hour day and night cycles at $25^{\circ} \mathrm{C}$ and $22^{\circ} \mathrm{C}$ temperatures, respectively. The plants were allowed to grow at $65 \%-70 \%$ relative humidity until harvest after 4 weeks.

\subsubsection{Generation of Modified Microcrystalline Cellulose (MMC) and Preparation of Affinity Columns}

Two grams of microcrystalline cellulose was suspended in $10 \mathrm{~mL}$ of double de-ionized distilled water ( $\mathrm{ddw})$, and mixed with $24 \mathrm{~mL}$ of $\mathrm{H}_{3} \mathrm{PO}_{4}(85 \%$ commercial grade) by constant stirring at room temperature for $30 \mathrm{~min}$ at a $60 \%$ calculated concentration of phosphoric acid used. The mixture was centrifuged at $1300 \mathrm{rpm}$ for $10 \mathrm{~min}$ at $4^{\circ} \mathrm{C}$. The obtained pellet was re-suspended in $30 \mathrm{~mL} \mathrm{ddw}$ and washed after neutralizing the remained $\mathrm{H}_{3} \mathrm{PO}_{4}$ with $1 \mathrm{~mL}$ of $2 \mathrm{M} \mathrm{Na}_{2} \mathrm{CO}_{3}$ solution. After repeating the process twice, the obtained 
MMC pellet was re-suspended in $30 \mathrm{~mL}$ ddw and adjusted at pH 7.0 with $2 \mathrm{M} \mathrm{Na}_{2} \mathrm{CO}_{3}$ drops. Finally, the suspension was stored at $4^{\circ} \mathrm{C}$, at $8 \mathrm{mg} / \mathrm{mL}$ MMC by matching a $250 \mathrm{~mL}$ volume up with ddw. The MMC suspension was packed directly into a BIO-RAD Econ-Column ${ }^{\circledR}(2 \mathrm{~cm} \times 20 \mathrm{~cm})$. For maintaining the constant flow of liquid, the translucent column was assembled with a Pharmacia LKB P-1 Laboratory Pump (Sweden) through capillary tubing connected with a porous polymer bed support fitting at the bottom of the column. The excess water of cellulose-slurry was pumped out @1 $1 \mathrm{~mL} / \mathrm{min}$ before drying the prepared resin beds of the column.

\subsubsection{Extraction of Recombinant Proteins}

The plant biomasses were crushed into powder in liquid nitrogen with a mortar and pestle and extracted in ice-cold binding buffers (BB). To enhance the release of intracellular proteins in the buffer, the mixture (on an ice bucket) was passed through $3 \times 100$ s pulses sonication by the BRANSON SONIFIER (Model 450, USA) set at a $30 \%$ duty cycle and $60 \%$ amplitude. The obtained homogenates were clarified by centrifugation at $2000 \times \mathrm{g}$ for $20 \mathrm{~min}$ at $4^{\circ} \mathrm{C}$. The supernatant was separated from the pelleted plant debris for affinity purification of the recombinant proteins.

\subsubsection{Affinity Separations}

\section{I. $\mathrm{FGF}_{1}$-CBD Tagged Protein}

The clarified extract of transient Nicotiana benthamiana leaves $(1.5 \mathrm{~g}$ and $2.0 \mathrm{~g})$ were prepared in $15 \mathrm{~mL}$ and $10 \mathrm{~mL}$ binding buffers (50mM NaPi - pH $6.5 ; 50 \mathrm{mM} \mathrm{NaCl} ; 1 \mathrm{mM}$ $\mathrm{CaCl}_{2} ; 1 \mathrm{mM}$ Phenylmethylsulphonyl fluoride (PMSF); $0.5 \%$ Triton X-100 and 50mM Tris - pH 7.0; $150 \mathrm{mM} \mathrm{NaCl} ; 1 \mathrm{mM}$ $\mathrm{CaCl}_{2} ; 1 \mathrm{mM}$ Phenylmethylsulphonyl fluoride (PMSF); $0.5 \%$ Triton X-100), respectively. The column was fed with the supernatant after equilibrating the resin bed with the binding buffer. At the end of affinity-run the resin was washed three times by agitating the bed with $10 \mathrm{~mL}$ of wash buffer $(20 \mathrm{mM}$ Na-Ac. pH 4.0; $50 \mathrm{mM} \mathrm{NaCl} ; 1 \mathrm{mM} \mathrm{CaCl} 2 ; 1 \mathrm{mM}$ PMSF) in each instance. Finally, the protein was released by mixing cellulose with three $2 \mathrm{~mL}$ aliquots of $50 \mathrm{mM}$ Tris $(\mathrm{pH} 8.0)$; $150 \mathrm{mM} \mathrm{NaCl} ; 1 \mathrm{mM} \mathrm{CaCl}_{2} ; 1 \mathrm{mM}$ PMSF, and $1 \%$ Cellobiose.

\section{II. $\mathrm{H}_{5} \mathrm{~N}_{1}-\mathrm{CBD}$ Fused Protein}

The transgenic Arabidopsis thaliana plant (1.0g) extract was clarified from $30 \mathrm{~mL}$ of binding buffer made from $10 \mathrm{mM}$ Tris ( $\mathrm{pH} 8.0), 150 \mathrm{mM} \mathrm{NaCl}, 1 \mathrm{mM} \mathrm{CaCl} 2,1 \mathrm{mM} \mathrm{PMSF}, 0.1 \%$ Triton $\mathrm{X}-100$. The total protein extract was loaded into the affinity column, saturated with the binding buffer. The column was washed five times, with $6 \mathrm{~mL}$ of binding buffer and occasional agitation of the resin bed. The immobilized protein was eluted using $2 \mathrm{~mL} 2 \%$ Triethylamine (SigmaAldrich, USA) solution thrice, followed by dilution of the elutes with equal volume of binding buffer without detergent. At every stage of the affinity separation process, the column was positioned vertically after settling the resins to form a clear interface with the total protein extracts, which were loaded at $4^{\circ} \mathrm{C}$. During fractionation of samples, the pump was set with constant flow rates of 100,500 , and $300 \mu \mathrm{L} / \mathrm{min}$ for adsorption, washing, and elution of fusion proteins, respectively.

\subsubsection{Assay of Proteins}

The recombinant proteins were quantified using a Bio-Rad dye-based assay reagent (Bio-Rad Laboratories, Inc. Hercules, CA 94547). The absorbance (at $595 \mathrm{nM}$ ) reading was taken by the TECAN SpectraFluor Plus multi-label micro plate reader installed with Magellan 3 software and data station. For quantitative assessment of proteins, a calibration curve with known concentrations of bovine serum albumin (BSA) as the authentic standard was considered. The apparent purity of proteins was verified by the SDS-PAGE gels stained with Coomassie Brilliant Blue R Staining Solution (Power Stain TM, ELPIS BIOTECH, Korea), followed by destaining with a solution $(7: 2: 1 ; \mathrm{MeOH}$ : acetic acid: water) prepared in our laboratory. The image of the gel was captured by Magic Scan 32 software v4.5 in UMAX PowerLook 1100 imaging system.

\subsubsection{SDS-Polyacrilamide Gel Electrophoresis, Immunoblotting}

The fractionated proteins were separated by SDS-PAGE with 12,10 , and $8 \%$ polyacrilamide levels (whenever necessary) and transferred onto nitrocellulose membranes (by semi-dry transfer unit; Hoefer ${ }^{\mathrm{TM}}$ TE 77, Amersham Biosciences) saturated with a $0.1 \%$ SDS transfer buffer (250mM Tris-base, 190mM Glycine and 20\% Methanol). The membranes were blocked in 3\% skimmed milk in TBS buffer containing $0.1 \%$ Tween 20 (TBS-T buffer) for $15 \mathrm{~min}$. An antibody, specific to the CBD raised in rabbits, diluted $(1: 100,000)$ in blocking buffer was used to incubate the membrane at $4{ }^{\circ} \mathrm{C}$ overnight. For detection, the membranes were rinsed three times every $10 \mathrm{~min}$ with TBS-T buffer. A secondary incubation of the membranes with a rabbit antibody (1: 10,000) was conducted at room temperature for 1 hour, prior to detection of signals on X-ray films (AGFA, Medical X-Ray film, blue, Agfa Health Care NV, Belgium).

\section{Results}

The antigenic proteins tagged with $\mathrm{CBD}$ domain, as represented in Figure 1 (A-B), were grown in plants and isolated through the affinity column packed with MMC. Out of the two versions (A and B) of protein cassettes, the Ctagged domain resulted in the production of recombinant protein of interest in Arabidopsis plants as of immunoblot signal in Figure 1 (C). The shift of CBD domain from Cterminus to $\mathrm{N}$-terminus of the construct failed to accumulate the target protein in plants (data not shown in Figure $1 \mathrm{C}$ ). Therefore, further experiments with $\mathrm{N}$-terminus fused $\mathrm{CBD}$ cassette were abandoned. Thus, it clearly demonstrates the functionality of the C-terminus CBD-linked construct for biosynthesis of recombinant proteins in plants as a production platform. 

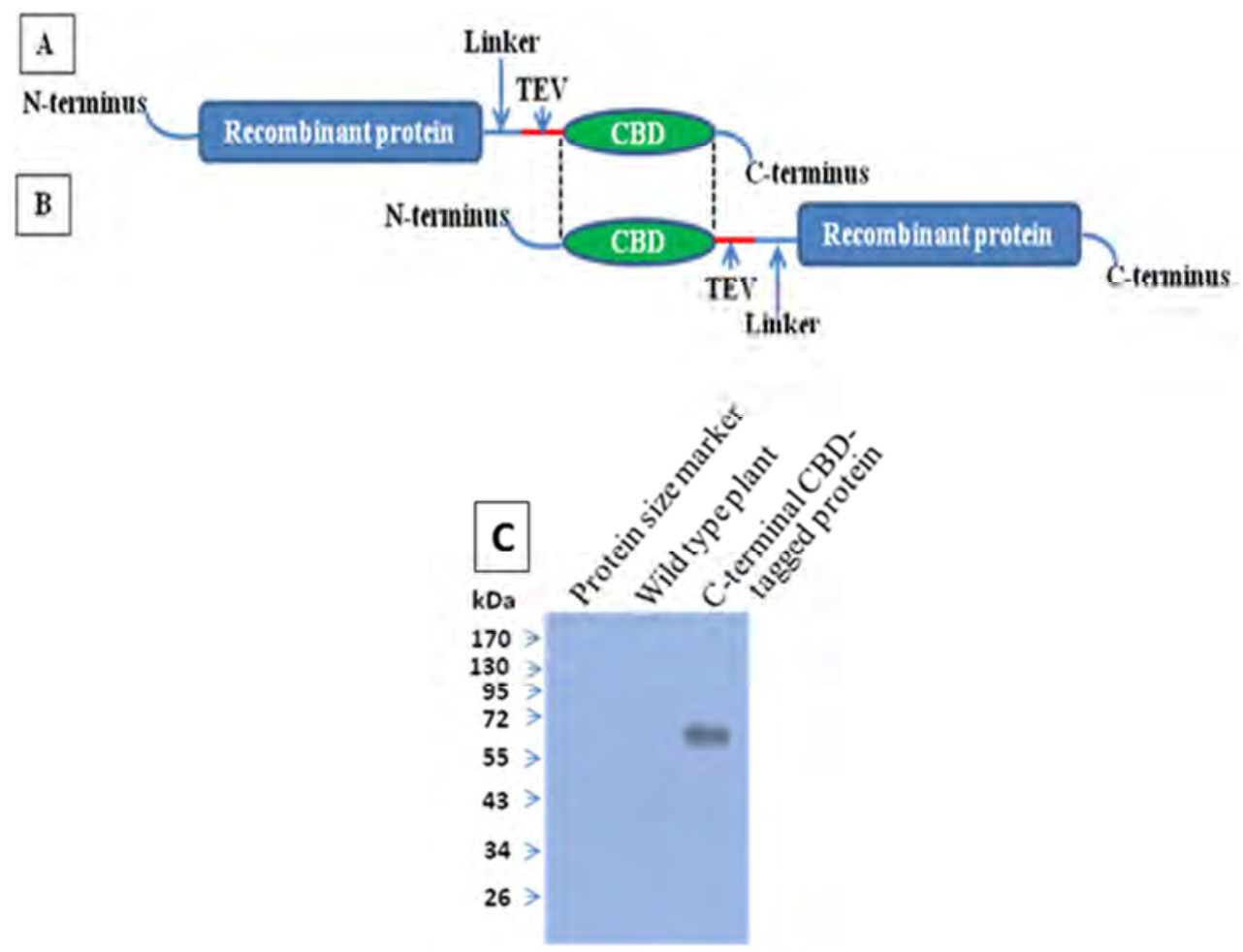

Figure 1. The fusion protein construct with CDB tag and the immunoblot of proteins translated in plant. $(A-B)$ Generalized view of domain constructs for the expression of fusion proteins in plants. CBD domains were shown on either of the C-terminus (A) and N-terminus (B) ends of the hypothetical fusion proteins. The linker regions and the Tobacco Etch Virus (TEV) as restriction enzyme sites for removing the tag from purified proteins were shown by down and upward arrows in $(A$ and $B)$ schemes respectively. (C) Immunoblot analysis of the crude extracts of TSP from wild type and transgenic Arabidopsis plants expressing the C-terminal CBD tagged insert were separated on a 10\% SDS-PAGE gel. Molecular masses of protein size marker are presented in $k D a$. The second lane contains the wild type protein used as a negative control of the analysis. The transgenic protein (ca. 58kDa) was detected by the immunoblot with CBD specific antibody (rabbit) as shown in the last lane from left.

\subsection{MMC-Affinity Purification of FGF ${ }_{1}-\mathrm{CBD}$ Fusion Protein}

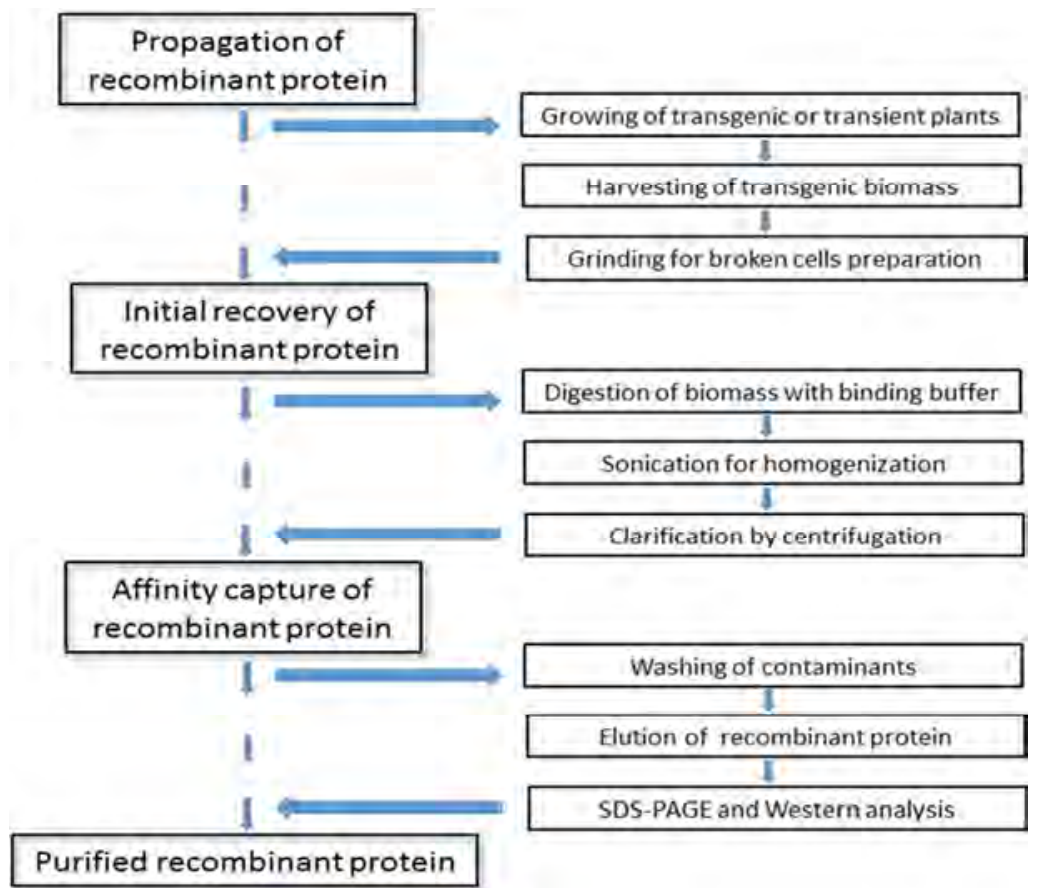

Figure 2. Purification strategy employed to investigate the downstream processing factors for purity of recombinant proteins in plants by cellulose affinity separation module. 
All the homogenates of fusion proteins under study were purified following the strategy depicted in the box flow diagram (Figure 2). In this study, the purification schemes have been optimized on a case-by-case basis of recombinant proteins engineered in either $N$. benthamiana or A. thaliana. When the sample was loaded in the presence of MMC presaturated with the binding buffers of ca. $\mathrm{pH} 7.0$ or $\mathrm{pH} 6.5$, the CBD fused proteins apparently adsorbed while passing across the column bed and the unbound proteins including the host proteins accumulated as unbound fractions. However, out of the two $\mathrm{pH}$ levels of binding buffers, the neutral $\mathrm{pH}$ facilitated better coupling of fusion proteins with cellulose (compare the intensities of immunoblot signals of unbound fractions on Figure 3A, Figure 3C). Many of the tagged proteins were un-trapped by the column resin and eventually went down to the unbound fraction collected during affinity binding with the buffer at $\mathrm{pH} 6.5$ (Figure 3C). Next, the washing of the column with decreased ionic strength $(20 \mathrm{mM})$ by $\mathrm{NaCl}$ at low $\mathrm{pH}(4.0)$ indicated zero loss of target proteins as evidenced by no signal through immunoblots of wash fractions $\left(\mathrm{W}_{1}\right.$ and $\mathrm{W}_{3}$ lanes in both panels of Figure 3A, and 3C). Thus, it suggests that a washbuffer with low $\mathrm{pH}$ and low ionic strength would be the best condition of choice for safe cleansing of column resins contaminated with non-specific native proteins co-extracted from the host plant.
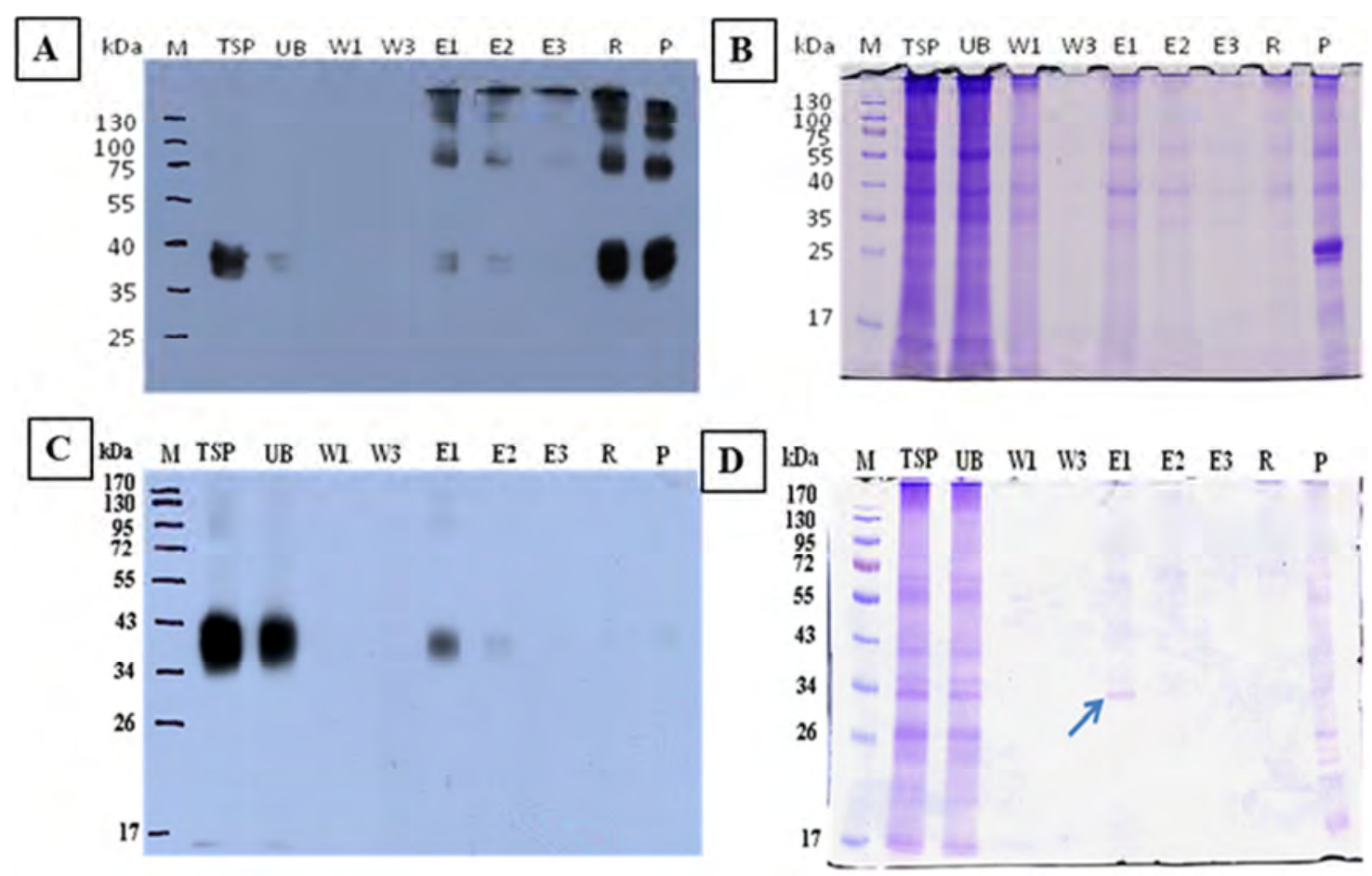

Figure 3. Western blot hybridization analysis of the proteins after fractionation of the crude extract obtained from transient $N$. benthamiana. (A) Proteins were resolved by $12 \%$ SDS-PAGE and probed with anti-CBD primary antibody (Rabbit). (B) SDS-PAGE analysis after fractionation of the crude tobacco (N. benthamiana) extract at 1:5 tissue-to-buffer ratio. Protein bands were stained with Coomassie blue after resolving by $12 \%$ SDS-PAGE gel. (C) Western blot hybridization analysis of the proteins after fractionation of the crude extract obtained from transient N. benthamiana. Proteins were separated by $12 \%$ SDS$P A G E$ and blotted and analyzed with anti-CBD primary antibody (Rabbit). (D) SDS-PAGE analysis after fractionation of the crude tobacco (N. benthamiana) extract at 1:10 tissue-to-buffer ratio. Protein bands were stained with Coomassie blue after resolving by 12\% SDS-PAGE gel. Annotations: M- protein size markers with their sizes indicated in kilo Dalton on the left of the pictures. TSP-total soluble proteins, UB- unbound, $W_{1-}$ first wash fraction, $W_{3}-$ third wash fraction, $E_{1^{-}}$first elution fraction, $E_{2}$ second elution fraction, $E_{3^{-}}$third elution fraction, $R$ - resin boiled fraction, $P$ - pellet fraction, both of the resin and pellet fractions were dissolved with equal volume of the extraction buffer.

Affinity elutions of the protein by $1 \%$ Cellobiose in Trisbase salt buffers ( $\mathrm{pH} 9.5$ and 8.0) are shown in Figure 3 (AB) and Figure 3 (C-D), respectively. The buffer (50mM Tris ( $\mathrm{pH}$ 9.5), $150 \mathrm{mM} \mathrm{NaCl}, 1 \mathrm{mM} \mathrm{CaCl} 2$ ) with $1 \%$ Cellobiose resulted in very little elution of the bound protein and thus faint signals were detected on the Western blot, but with no visible band at the corresponding position of the gel stained in Coomassie blue (lanes $E_{1}, E_{2}, E_{3}$ for elution fractions in Figure $3 \mathrm{~A}$ and $3 \mathrm{~B}$ ). Consequently, the great majority of the proteins of interest were detected in the resin fraction on the same gel ( $\mathrm{R}$, resin boiling lane in Figure $3 \mathrm{~A})$. However, a perfect elution of the $\mathrm{CBD}$-fused $\mathrm{FGF}_{1}$ protein was conducted by the same composition of buffer but at pH 8.0. The elution yield was maximized by the first run of the buffer in comparison to the other elution fractions (compare lanes $E_{1}, E_{2}$, and $E_{3}$ of Figure $3 C$ and Figure $3 D$ ). The purified $\mathrm{FGF}_{1}-\mathrm{CBD}$ protein showed near homogeneity and was barely visible, indicating little, if any, protein contamination in the first elution sample loaded in the Coomassie stained SDSPAGE gel ( $E_{1}$ lane of Figure 3D). This result was supported by the corresponding Western blot signal ( $E_{1}$ lane) in the Figure 3C.

At the beginning of our study, the clarified extract was generated from the concentrated tobacco-slurry prepared with 
1:5 tissue-to-buffer ratio. As such, many of the target proteins escaped into the pelleted plant debris ( $\mathrm{P}$ lane of Figure 3A). Contrastingly, the digestion mixture, prepared at 1:10 tissueto-buffer ratio, turned out with no residue at the pellet fraction as detected on the P-lane of Figure 3C. Thus, the initial recovery of recombinant protein was much more efficient in diluted digestion of plant tissues over the concentrated one. The result (shown in Figure 3C) clearly demonstrates an optimized option ensuring the significant initial recovery without any loss of target molecules, which facilitates diversion of the maximum amount of target molecules into the affinity separation streaming for higher yield of recombinant proteins.

\subsection{Initial Recovery and Isolation of $\mathrm{H}_{5} \mathrm{~N}_{1}$-CBD Protein}

One of the major targets of our research was to perform an initial recovery and capture on cellulose beads for concurrent isolation of the recombinant protein efficiently. As a partial proof of our initial concept, we successfully recovered the total $\mathrm{H}_{5} \mathrm{~N}_{1}-\mathrm{CBD}$ protein in Arabidopsis by extracting TSP twice from the crude homogenized slurry in the presence of a buffer at a tissue-to-buffer ratio 1:15 (Figure 4A P-lane). Manipulating this ratio at 1:10 resulted in inefficient recovery of fusion protein into the start solution. With this condition, a marginal fraction of the recombinant protein concomitantly separated with the tissue debris as detected by the immunoblot signal generated from the pellet sample (Figure 4C, P-lane). Thus, the dilution ratio (tissue-to-buffer) of slurry appeared as a key parameter in releasing the fusion protein into the clarified extract. In another study with the identical condition, except no repeat of extraction, the 1:15 dilution ratio of extraction resulted in incomplete recovery of the protein of interest (Figure 5A, P-lane). On the other hand, the diluted buffer $(1: 30)$ facilitated a complete initial recovery with extraction once followed by centrifugation of the homogenate (Figure 5B, P-lane).

The fusion protein was immobilized efficiently to the MMC-affinity column equilibrated with the buffer $(10 \mathrm{mM}$ Tris-base (pH-8.0), 150mM NaCl, $1 \mathrm{mM} \mathrm{CaCl} 2,1 \mathrm{mM}$ PMSF and $0.1 \%$ Triton-X100) (as of negligible loss verified by the defused signal of unbound fraction shown in UB lane of Figure 3C). As a comparison, a similar experiment set was conducted with an identical affinity column but using a submerged resinbed with binding buffer above. However, most of the CBDtagged protein was not captured onto the MMC and consequently they were detected as unbound and first wash fractions by immunoblot analysis (Figure 4A, UB and $\mathrm{W}_{1}$ lane). This result clearly indicates that the compact resin bed creates a favorable environment for adsorption-cum-binding of the fusion protein by the cellulose matrix (Figure 4C, UB lane) but not the loose bed due to the condition inundated by the buffer (Figure 4A, UB and $\mathrm{W}_{1}$ lanes).
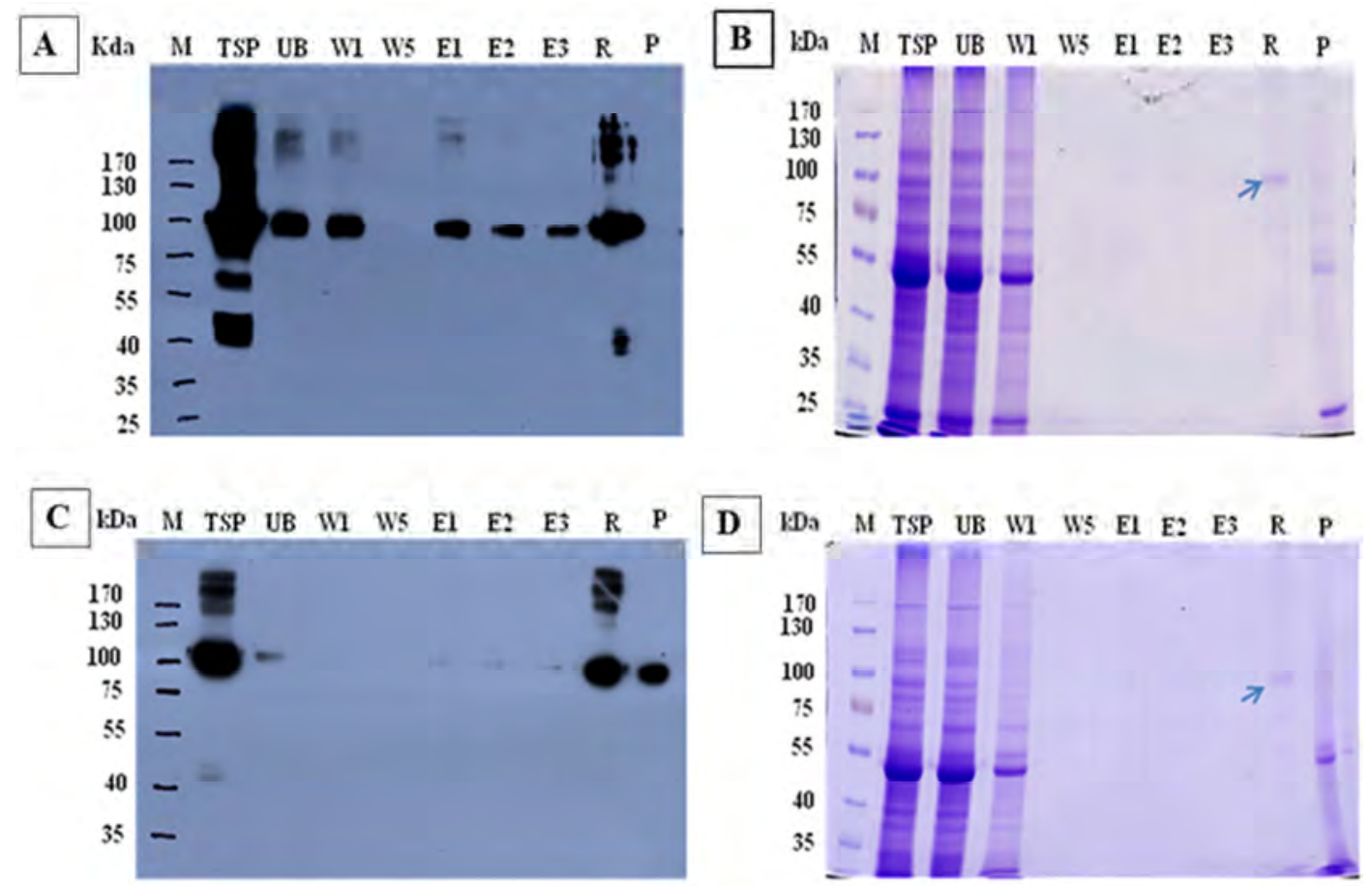

Figure 4. Western blot analysis with different tissue to buffer ratio. The SDS-PAGE of the samples were fractionated following the entire affinity purification scheme, hybridized with an antibody specific to the CBD tag raised in rabbit. The gels were prepared with $8 \%$ SDS-polyacrilamide preparation. The lanes correspond exactly to that shown in $M$, protein size standards $(\mathrm{kDa}) ; \mathrm{TSP}$, total soluble protein; UB, unbound fraction; $W_{1}$, first wash fraction; $W_{5}$, fifth wash fraction; $E_{1}$, first elution fraction; $E_{2}$, second elution fraction; $E_{3}$, third elution fraction; $R$, boiled-resin fraction; $P$, pellet (during clarification) fraction. (A) Resin and pellet fractions were diluted with same volume of extraction buffer. The slurry was prepared with 1: 15 tissue-to-buffer ratio. (B, D) The samples were fractionated following the entire affinity purification scheme, stained with Power Stain Coomassie blue. (C-D) Resin and pellet fractions were diluted with same volume of extraction buffer. The slurry was prepared with 1: 10 tissue-to-buffer ratio. 

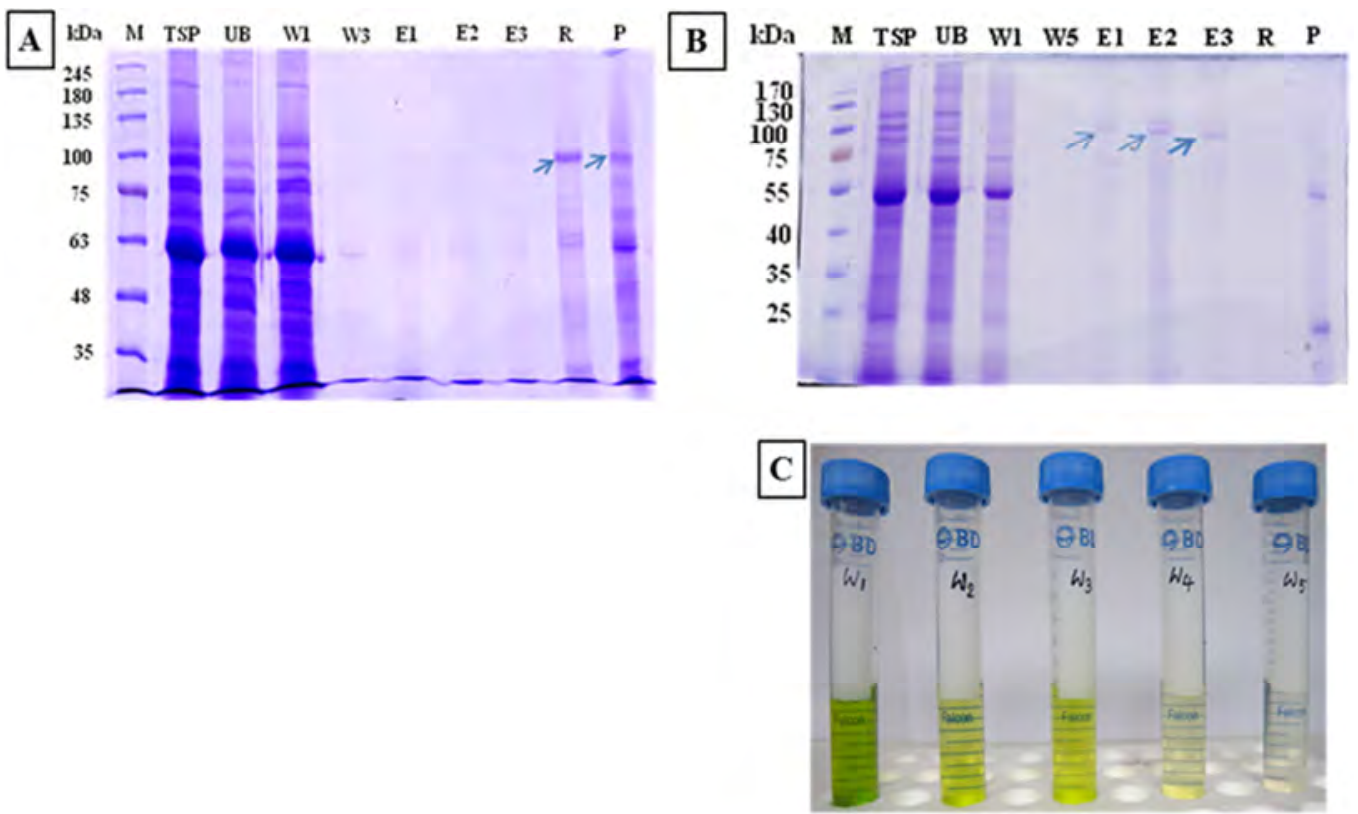

Figure 5. Coomassie blue stained SDS-PAGE (10\%) gel loaded with the fractions obtained during the purification of $H_{5} N_{I}$-CBD protein transgenically expressed in Arabidopsis. Lanes: $M$, protein size standards $(k D a)$; TSP, total soluble protein; UB, unbound fraction; $W_{1}$, first wash fraction; $W_{5}$, fifth wash fraction; $E_{1}$, first elution fraction; $E_{2}$, second elution fraction; $E_{3}$, third elution fraction; $R$, boiled-resin fraction; $P$, pellet (during clarification) fraction. Resin and pellet fractions were diluted with same volume of extraction buffer. The arrow marks denote the $H_{5} N_{1}-C B D$ fused proteins isolated through the affinity purification scheme proposed. (A) Dilution of slurry was made at 1:10 tissue-to-buffer ratio. (B) Proteins were visualized by 10\% SDS polyacrilamide gel stained with Coomassie brilliant blue. The loaded fractions were derived from the clarified solution from the Arabidopsis slurry (at 1: 30 tissue-to-buffer ratio). (C) Effect of washing frequencies on removal of host impurities by the fractions $\left(W_{1}\right.$ to $W_{5}$ ) collected during washing of the affinity column. Only the Arabidopsis pigments are visible in wash fractions above.

We observed that the binding buffer could be treated as a wash buffer. It did not have any effect on column washing of non-specific proteins. As such, the binding-cum-wash buffer supplemented with detergent $(0.1 \%$ Triton X-100) improved cleaning the contaminating proteins off without any loss of fusion protein immobilized onto the column resin (Figure 4C, $\mathrm{W}_{1}$ and $\mathrm{W}_{5}$ lane as verified here). The frequency and volume of washing dictated the purifying protein obtained. As demonstrated by the Coomassie stained SDS-PAGE gels in our study, the first wash removes a great majority of the native proteins of Arabidopsis from the affinity columns. However, multiple washes (upto five times) equivalent to the extraction volume of TSP facilitated in obtaining clarified column bed (data not shown) with immobilized protein released by boiling of cellulose beads ( $R$ lanes in Figure 4B and 4D). Contrastingly, a washing frequency limited to three times, with total volume less than TSP extraction buffer, proved to be an inefficient strategy, with huge amounts of contaminated proteins bound to the resin, along with the recombinant protein (Figure 5A, R-lane). Higher buffer volumes with frequencies improved washing efficiency in our protein purification scheme as also evidenced by the clarified resin and the decreased visual color intensities, with successive wash-fractions (Figure $5 \mathrm{C}$ ). The bound $\mathrm{H}_{5} \mathrm{~N}_{1}$ CBD protein was poorly released from the column with $1 \%$ Cellobiose prepared in $150 \mathrm{mM} \mathrm{NaCl}$ and $1 \mathrm{mM} \mathrm{CaCl}_{2}$ supplemented with either $10 \mathrm{mM}$ Tris-base $(\mathrm{pH} 8.0), 10 \mathrm{mM}$ Hepes (pH 7.5), or $1 \mathrm{mM}$ Tris-base (11.0) (Figure 4A, 4C, $4 \mathrm{D}, 5 \mathrm{~A}$; elution lanes $-\mathrm{E}_{1}, \mathrm{E}_{2}, \mathrm{E}_{3}$ in each of the figures). Nevertheless, the recombinant protein eluted successfully with $2 \%$ Triethylamine (TEA) accompanied by dilution of eluents with equal volume of binding buffer. The eluted protein was visualized with adequate level of purity on SDSPAGE gel stained with Coomassie Brilliant Blue dye $\left(\mathrm{E}_{1}, \mathrm{E}_{2}\right.$, and $\mathrm{E}_{3}$ lanes on Figure 5B).

\section{Discussion}

Molecular bioengineering technology has come of age, with the advent of the first plant-made recombinant protein in the market, and many more similar technologies in the pipeline [29]. As such, there is a need to explore the opportunity for efficient commercial production and purification of alien proteins for new therapeutics, disease diagnostics, and vaccine derivatives in plants. The purification of therapeutic proteins using plant systems present a number of advantages, including a cost-effective platform for large-scale production, and reduced safety concerns over the traditional mammalian and microbiological bio-reactor systems [30]. The overall cost of production of growing recombinant proteins in open-field crops, is clearly $30-40 \%$ less when compared to the bioreactor-based protein generation process, despite similar downstream purification costs in both systems [31]. The down-stream handling cost accounts significantly higher than the upstream propagation of foreign proteins in plants. Thus, our research focus concentrates on bottom areas to address overall productivity of the recombinant production of proteins in plants [32]. Additionally, there is a need for a simple, but cost-effective purification protocol that can be 
used a tool for the efficient handling of target proteins, expressed at a lower concentration in plants.

Thus, we developed a non-expensive but simple and reproducible method to purify vaccine antigens by focusing on two phases: (1) initial recovery and (2) the affinity capture of recombinant proteins during down-stream processing of the plant extracts. The primary objective of the initial recovery was to ensure the product titre of the clarified extract derived from the plant homogenate was at a maximum. We considered the product recovery phase as critical because it dictates the amount of new proteins, generated in the start material of plant biomass, to be released into the extract. The types of impurities with their corresponding amounts present issues and have to be cleaned during the handling of extract as reported elsewhere [32]. The physical conditions for primary recovery after biomass harvest from plants are similar in some operations such as crushing, centrifugation, clarification, although their strategic application towards separation of target molecules are defined by the extract composition, extract properties and product stability [33]. However, the primary recovery condition essentially varies with tissue type (e.g., leaves, seeds, roots) and plant species. The composition of biological sources of foreign protein (e.g., plants) are normally contaminated by significant amounts of native compounds, such as proteins, lipids, pigments, and phenolic compounds, which could compromise the initial harvest of proteins [34].

The initial protein extraction or release process is critical because it relies on the total extract volume required to dissolve the TSP of homogenate. A variety of operational conditions were conducted by manipulating the extraction buffer at a tissue-to-solution ratio of $1: 13.3$ and $1: 2$ to $1: 20$, adjusted in order to purify the protein of interest in different sources of tissues [29, 31] but without information on the target proteins with plant debris as wastage. The choice of differential dilutions of the plant homogenate is dependent on several parameters such as the water content of biomass, oilbodies, and the concentration of recombinant proteins associated with host compounds as impurities to be removed during purification process [33]. The extraction and removal of oil-bodies requires much higher buffer to tissue ratios [35]. Additionally, the diluted slurry prepared from wellhomogenized tissues with disrupted cell walls help maximize the intracellular protein released into the solution, followed by the efficient isolation of the pelleted plant debris through centrifugation. Although the lower start volume of the digestion buffer reduces the process volume for purification, it represents a potential interfering factor during isolation process. In this case the main limitation could be raised by lowering the percentage of recombinant protein extracted [31] and by fouling of the chromatographic column used in later purification stages [36]. Consequently, the affinity immobilization and washing efficiency is decreased due to the concentrated plant pigments and cellular impurities adsorbed onto the cellulose-affinity- matrices of the column.

Cellulose is a widely accepted bio-matrix for separation of recombinant proteins tagged with cellulose-binding-domains because of its mechanical and chemical stability, costeffectiveness for large-scale operations, and ability to handle large volumes [37]. Notably, cellulosics are declared safe for many pharmaceutical and human uses [38]. Cellulose is also suitable for downstream processing of recombinant proteins with therapeutic properties. Commercial microcrystalline cellulose is usually considered as a coupling partner for affinity purification of proteins fused with CBD. Regenerated Amorphous Cellulose (RAC), a derivative of microcrystalline cellulose, has high binding capacity of proteins compared to other celluloses available in the market [39]. Despite the obvious advantage of RAC in affinity binding, however, it causes blocking of the affinity column (data not shown) and thus is not suitable for successive operations such as washing and elution of proteins, unless the column has been spiked with a reasonable amount of microcrystalline cellulose to increase the porosity of the resin bed. In this perspective the MMC is very efficient both in adsorption of tagged proteins with subsequent wash ability due to sufficient bed-porosity of the affinity column as well.

Efficient purification of biomedical proteins grown through heterologous expression in plants is a major challenge. This challenge is primarily complicated due to low yield (compare to microbial systems) of target proteins with concomitant contamination due to compounds released from the feed material of plants. In this study, we developed a new but simple method for purifying CBD-tagged vaccine antigens utilizing non-expensive cellulose (modified) beads as a specific adsorbent in the affinity column. Commercial microcrystalline cellulose has some interesting properties as an excellent affinity substrate [40]. Nevertheless, we utilize $\mathrm{MMC}$, as a newly regenerated adsorbent, taking advantage of its high capacity to trap target proteins, with removal of impurities through intensive washing of resin, resulting in almost no leakage of fusion proteins.

\section{Conclusion}

The use of CBD affinity tail is demonstrated as a functional domain co-expressed with antigenic proteins in plants. This technique allows us to isolate the engineered proteins from a new and easy procedure, unlike those currently employed for affinity purification of plant proteins, requiring multipurification steps and expensive chromatographic columns. Although we did not pay attention to the quantitative values of the proteins engineered in plants, we monitored the target proteins critically by optimizing the conditions to reduce potential losses through fractions collected and their subsequent verification by the immunoblots specific to the CBD antibody. As such, this optimized method has the potential to be scaled up to produce fusion proteins on a preparative scale, using inexpensive MMC resin and buffers made of salts, water and other additives.

\section{Acknowledgements}

The authors thanks for the financial assistances for the 
research offered by the National Research Foundation of Korea (NRF) grant funded by the Korea government (MEST) (2014R1A2A2A01002931) and the Next-Generation BioGreen 21 Program, Rural Development Administration, Republic of Korea (PJ01121601).

\section{References}

[1] Chan H. T., Daniell, H., 2015. Plant-made oral vaccines against infectious diseases - Are we there yet? Pl. Biotech. J.13: 1056-1070.

[2] Korban S. S., 2002. Targeting and expression of antigenic proteins in transgenic plants for production of edible vaccines. In vitro Cellular and Developmental Biol.-Plant 38: 231-236.

[3] Wycoff K. L., 2005. Secretory IgA antibodies from plants. Current Pharmaceutical Design 11: 2429-2437.

[4] Fischer R., Schillberg S. (Eds.) 2004. Molecular Farming: Plant-made Pharmaceuticals and Technical Proteins WILLEY-VCH Verlog GmbH \& Co., KGaA, Weinheim.

[5] Streatfield J., Howard J. A., 2003. Plant Production Systems for Vaccines. Expert Review of Vaccines 2: 763-775.

[6] Fiske M. J., Fredenburg R. A., VabDerMeid K. R., McMichael J. C., Arumugham R, 2001. Method for Reducing Endotoxin in Moraxella catarrhalis UspA2 Protein Preparations, $J$. Chromatogr. B Biomed. Sci Appl.753:269-278.

[7] Magalhaes P. O., Lopes A. M., Mazzola P. G., Rangel-Yuagui C., Penna T. C., Pessoa A. Jr., 2007. Methods of Endotoxin Removal from Biological Preparations, a review, J. Pharm. Pharm. Sci.10: 388-404.

[8] Hirayama C., Sakata M., 2002. Chromatographic removal of endotoxin from protein solutions by polymer particles. $J$. Chromatogr. B Analyt. Technol. Biomed. Life Sci.781: 419-432.

[9] Herbes K., Sonnewald U., 1999. Production of new / modified proteins in transgenic plants. Current Opin. in Biotech. 10: 163-168.

[10] Conard U., Fiedler U., 1998. Compartment specific accumulation of recombinant immunoglobulins in plant cells: an essential tool for antibody production and immunomodulation of physiological functions and pathogen activity. Pl. Mol. Biol. 38: 101-109.

[11] Arntzen C. J., 1998. Pharmaceutical foodstuffs-oral immunization with transgenic plants. Nat. Med. Vaccine Suppl. 4: 502-503.

[12] Schillberg S., Emans N., Fischer R., 2002. Antibody molecular farming in plants and plant cells. Phytochem. Rev.1: 45-54.

[13] Stoger, E., Sack, M., Fischer, R., Christou, P., 2002. Plantibodies: applications, advantages and bottlenecks. Curr. Opin. Biotechnol.13(2):161-166.

[14] Stoger, E., Sack, M., Nicholson L., Fischer, R., Christou, P., 2005. Recent Progress in Plantibody Technology. Current Pharma. Design11: 2439-2457.

[15] Hellwigs S., Drossard J. Twyman R. M., Fischer R., 2004. Plant cell cultures for the production of recombinant proteins. Nature Biotech. 22(11): 1415-1422.
[16] Smith D. B., Johnson, K. S., 1998. Single-step purification of polypeptides expressed in Escherichia coli as fusions with glutathione S-transferase. Gene 67: 31-40.

[17] Hedouelle, H., Duplay, P., 1998. Production in Escherichia coli and one-step purification of bifunctional hybrid proteins which bind maltose. Export of the Klenow polymerase into the periplasmic space. Eur. J. Biochem.17: 541-549.

[18] Porath, J. Carlsson, J., Olsson, I., Belfrage, G., 1975. Metal Chelate affinity Chromatography, a new approach to protein purification. Nature 258: 598-599.

[19] Lichty, J. J., Malecki, J. L., Agnew, H. D., MichelsonHorowitz, D. J., Tan, S., 2005. Comparision of affinity tags for protein purification. Protein Express. Purif. 41: 98-105.

[20] Shani, Z., Shoseyov, O., 2001. Process of expressing and isolating recombinant proteins and recombinant protein products from plants, plant derived tissues or cultured cells. US patent 6: 331- 416 .

[21] Shoseyov, O., Shani, Z., Levy, I., 2006. Carbohydrate binding modules: biochemical properties and novel applications. Microbiol. Mol. Biol. Rev. 70(2): 283-95.

[22] Rodriguez, B., Kavoosi, M., Koska, J., Creagh, A. L., Kilburn, D. G., Haynes, C. A. 2004. Inexpensive and generic affinity purification of recombinant proteins using a family 2a CBM fusion tag. Biotechnol. Prog. 20(5): 1479-89.

[23] Hearn, M. T. W., Acosta, D., 2001. Applications of novel affinity cassette methods: Use of peptide fusion handles for the purification of recombinant proteins. J. Mol. Recognit. 14: 323-369.

[24] Evangelista, R. L., Kusnadai, A. R., Howard, J. A., Nikolov, Z. L., 1998. Process and economic evaluation of the extraction and purification of recombinant beta-glucoronidase from transgenic corn. Biotechnol Prog. 14: 607-614.

[25] Stoger, E., Sack, M., Nicholson, L. Fischer, R., Christou, P., 2005. Recent progress in plantibody technology. Curr. Pharm. Design 11: 2439-2457.

[26] Witcher, D., Hood, E. E., Peterson, D., Baily, M., Bond, D., Kusandai, A., Evangelista, R., Nikolov, Z., Wooge, C., Mehigh, R., Kappel, W. Register, J. C., Howard, J. A., 1998. Commercial production of $\beta$-glucoronidase (GUS): a model system for the production of protein in plants. Mol. Breed. 4: 301-312.

[27] Conard, U., Fiedler, U. 1998. Compartment-specific accumulation of recombinant immunoglobulins in plant cells: as essential tool for antibody production and immunomodulation of physiological functions and pathogen activity Pl. Mol. Biol. 38: 101-109.

[28] Voinnet O, Rivas S, Mestre P, Baulcombe D., 2003. An enhanced transient expression system in plants based on suppression of gene silencing by the $\mathrm{p} 19$ protein of tomato bushy stunt virus. Pl. J. 33: 949-956.

[29] Platis, D., Drossard, J., Fischer, R., Ma, J. K.-C., Labrou, N. E., 2008. New downstream processing strategy of the purification of monoclonal antibodies from transgenic tobacco plants. J. Chromatogr. A: 80-89.

[30] Fischer, R., Emans, N., 2000. Molecular farming of pharmaceutical proteins. Transgenic Res. 9: 279-299. 
[31] Wilken, L. R., Nikolov, Z. L., 2012. Recovery and purification of plant-made recombinant proteins. Biotechnol. Adv. 30: 419433.

[32] Gottschalk, U., 2008. Bioseparation in antibody manufacturing: the good: the bad and the ugly. Biotechnol. Progr. 24: 496-503.

[33] Nicolov, Z. L. Regan, J. R., Dcikey, L. F., Woodard, S. L., 2009. Purification of antibodies from transgenic plants. In: Gottschalk U., editor. Process scale purification of antibodiesWiley-VCH:387-406.

[34] Barros, G. O. F., Woodard, S. L., Nikolov, Z. L., 2011. Phenolics removal from transgenic Lemma minor extracts expressing $\mathrm{mAb}$ and impact on $\mathrm{mAb}$ production cost. Biotechnol. Progr. 27: 410-418.

[35] Nykiforuk, C. L., Shen, Y., Murray, E. W., Boothe, J. G., Bussenil, D. et al., 2011. Expression and recovery of biologically active recombinant Apolipoprotein $\mathrm{Al}$ Milano from transgenic safflower (C. tinctorius) seeds. Pl. Biotechnol. 9: 250-263.
[36] Holler, C., Zhang, C., 2007. Purification of an acidic recombinant protein from transgenic tobacco. Biotechnol. Bioeng. 99(4): 902-909.

[37] Hong, J., Ye, X., Wang, Y., Zhang, Y.-H. P., 2008. Bioseparation of recombinant cellulose-binding moduleproteins by affinity adsorption on an ultra-high-capacity cellulose adsorbent. Analytica Chemica Acta 621: 193-199.

[38] Varela, M. Kimmel, P. L., Phillips, T. M., Mishkin, G. J. Lew, S. Q., Bosch, J. P., 2001. Biocompatibility of hemodialysis membranes: interrelations between plasma complement and cytokine levels. Blood Purif. 19:370-379.

[39] Hong, J., Wang, Y., Ye, X., Zhang, Y.-H. P., 2008. Simple protein purification through affinity adsorption on regenerated amorphous cellulose followed by intein self-cleavage. $J$. Chromatogr. A: 150-154.

[40] Shoseyov, O., Shani, Z., Levy, I., 2006. Carbohydrate Binding Modules: Biochemical Properties and Novel Applications. Microbiol. Mol. Biol. Rev. 70 (2):283- 295. 